\title{
LncRNA SNHG 6 drives proliferation and invasion of papillary thyroid cancer through modulation of miR-497
}

This article was published in the following Dove Medical Press journal:

OncoTargets and Therapy

\author{
Qiang Wen ${ }^{1, *}$ \\ Lina Zhao ${ }^{2, *}$ \\ Tongtong Wang' \\ Ningning $L v^{\prime}$ \\ Xuejiao Cheng' \\ Guang Zhang ${ }^{2}$ \\ Lin Bai'
}

'Department of Nuclear Medicine, China-Japan Union Hospital of Jilin University, ErDao District, Changchun 13033, China; '2Department of Thyroid Surgery, China-Japan Union Hospital of Jilin University, ErDao District, Changchun I3033, China

*These authors contributed equally to this work
Correspondence: Guang Zhang; Lin Bai China-Japan Union Hospital of Jilin University, I26\# Xiantai Street, ErDao District, Changchun I3033, China Tel +86 431 84995999

Email zhangguang_74@I63.com; linbai830@tom.com
Background: Long noncoding small nucleolar RNA host gene 16 (SNHG16) has been shown to play an oncogenic role in multiple cancers. However, the biological roles and mechanism of SNHG16 action in the regulation of papillary thyroid cancer (PTC) remains unknown. The aims of this study were to investigate the roles and the possible mechanism of SNHG16 in PTC progression.

Materials and methods: The expression of SNHG16 PTC tissues and cell lines was detected by reverse-transcription quantitative PCR (qRT-PCR). The effect of SNHG16 on cell proliferation, apoptosis, migration, and invasion was detected by Cell Counting Kit-8, flow cytometry, woundhealing assay, and Matrigel invasion assay, respectively. In addition, the regulatory relationships between SNHG16 and miR-497 were explored by luciferase reporter assay and qRT-PCR.

Results: The SNHG16 expression was upregulated in PTC tissues and cell lines, whose expression was positively associated with advanced TNM stage and lymph node metastasis. Function analysis demonstrated that depletion of SNHG16 in PTC cells significantly inhibited cell proliferation, induced cell apoptosis, and suppressed cell migration and invasion abilities. Mechanistic studies indicated that SNHG16 functioned as an endogenous sponge for miR-497 to regulate its target genes brain-derived neurotrophic factor and yes-associated protein 1 expression. Furthermore, the inhibition of miR-497 antagonized the suppressive effect of SNHG16-depleted cells on cell proliferation, migration, and invasion.

Conclusion: These findings revealed that SNHG16 drived the PTC progression possibly via regulating miR-497, suggesting that SNHG16 might be a novel therapeutic agent for PTC.

Keywords: papillary thyroid cancer, long noncoding RNAs, SNHG16, miR-497

\section{Introduction}

Thyroid cancer is the most common endocrine malignancy whose incidence has been markedly increasing worldwide in the past decade. ${ }^{1}$ Papillary thyroid cancer (PTC), accounting for more than $80 \%$ of all thyroid cancers, is the main histologic type of thyroid cancer. ${ }^{2}$ Despite the fact that patients with PTC have good prognosis and low mortality rate, about $15 \%$ of patients show aggressive behaviors and progress to poor outcome. ${ }^{3,4}$ Hence, there is an urgent need to understand the molecular mechanisms of PTC for developing diagnosis marker and targeted therapy for PTC.

Long noncoding RNAs (lncRNAs) are functionally defined as transcripts longer than 200 nucleotides in length that are unlikely to be translated into proteins. ${ }^{5}$ A growing body of evidence has indicated that lncRNAs play critical regulatory roles in physiological and pathological processes. ${ }^{6}$ LncRNAs are frequently dysregulated in various cancers and implicated in tumor development and progression. ${ }^{7,8}$ A number 
of lncRNAs have been identified to be involved in initiation and development of PTC, and function as oncogene or tumor suppressor in PTC progression, ${ }^{9,10}$ suggesting that lncRNAs could serve as diagnosis markers and therapy target for this disease. Therefore, identification of key lncRNAs involved in the PTC progression is of significance.

Small nucleolar RNA host gene 16 (SNHG16), a noncoding RNA, was reported to be upregulated and function as an oncogene that promoted tumorgenesis and tumor progression in multiple cancers, such as esophagus cancer, ${ }^{11}$ cervical cancer, ${ }^{12}$ bladder cancer, ${ }^{13}$ glioma, ${ }^{14}$ ovarian cancer,${ }^{15}$ gastric cancer, ${ }^{16}$ breast cancer, ${ }^{17}$ and colorectal cancer. ${ }^{18}$ Although recently a study showed that SNHG16 might be used to discriminate thyroid cancer from benign thyroid nodules, ${ }^{19}$ the role and the potential regulatory mechanism of SNHG16 in the PTC progression remain unknown.

Accumulating evidence suggested that lncRNAs act as endogenous miRNA sponges that abolish the effect of these miRNAs by interfering with the miRNA pathway, functioning as competing endogenous RNAs (ceRNAs). ${ }^{20}$ This lncRNA-miRNA regulatory network has been reported to play crucial roles in tumor progression in various human cancers. ${ }^{21}$ miR-497 has been reported to function as tumor suppressor in various cancers including PTC. ${ }^{22,23}$ However, cross-regulation between SNHG16 and miR-497 remained unclear. The aims of the current study, therefore, were to explore the roles of SNHG16 in PTC and investigate the association between SNHG16 and miR-497 to reveal the underlying regulatory mechanisms of PTC development.

\section{Materials and methods}

\section{Patients and samples}

A total of 48 PTC tissues and paired adjacent normal thyroid tissues were collected from patients with PTC who underwent radical surgical resections at China-Japan Union Hospital of Jilin University (Changchun, China). The patients included 32 women and 16 men, with an age range from 38 to 78 years and a mean of 57.0 years. None of the patients received radiotherapy or chemotherapy and any other therapy prior to surgery. All samples were confirmed by pathological examination in our hospital. All tissues were immediately frozen in liquid nitrogen after surgery and stored until RNA extraction. Written informed consent was obtained from patients with PTC. This study was conducted in accordance with the Declaration of Helsinki and was approved and supervised by the Ethical Committee of China-Japan Union Hospital of Jilin University.

\section{Cell cultures}

Three human PTC cell lines IHH-4, TPC-1, and HTH83, and normal thyroid follicular epithelial cell line Nthy-ori 3-1 were brought from Cell Center of Shanghai Institutes for Biological Sciences (Shanghai, China), and cultured as described previously. ${ }^{24}$

\section{Quantitative reverse-transcription PCR analysis}

Total RNA from tissues and cultured cells was extracted using TRIzol reagent (Thermo Fisher Scientific, Waltham, MA, USA) according to the manufacturer's instructions. Reverse-transcription reactions were conducted using a PrimeScript first-strand cDNA synthesis kit (Takara Biotechnology Co., Ltd., Dalian, China) following the manufacturer's protocol. cDNA was amplified using SYBR Premix ExTaq (Takara Biotechnology Co.) in the ABI 7900 fast system (Thermo Fisher Scientific). The primers used in this study are listed in Table 1. Relative quantitation of gene expression levels was calculated according to the $2^{-\Delta \Delta \mathrm{Cq}}$ method following normalization against U6 for miR-497 or GAPDH for $S N H G 16, B D N F$, and $Y A P 1$ mRNAs. All reactions were independently performed three times.

\section{Cell transfection with small interfering RNA (siRNA), mimic, or inhibitor} siRNA targeting SNHG16 (si-SNHG16, GGAAUGAA GCAACUGAGAUUU) and negative control scramble (siNC, UUCUCCGAACGUGUCACGUTT) were designed and synthesized by GenePharma (Shanghai, China). The miR-497 mimic and the appropriate negative control mimic (miR-NC), miR-497 inhibitor and corresponding control

Table I Real-time PCR primers used for mRNA or miRNA expression analysis

\begin{tabular}{l|l}
\hline Target gene & Primer $\left(\mathbf{5}^{\prime} \mathbf{- 3}^{\prime} \mathbf{)}\right.$ \\
\hline U6 & F-TCCGATCGTGAAGCGTTC \\
miR-497 & R-GTGCAGGGTCCGAGGT \\
& F-AGTCCAGTTTTCCCAGGAATCCCT \\
SNHGI6 & R-ACCAGCAGCACACTGTGGTTTGT \\
BDNF & R-TGTTCGTCATGGGTGTGAAC \\
& F-ATGGCATGGACTGTGGTCAT \\
YAPI & R-AGTGGTGGTCT GAGGTTGG \\
GAPDH & F-AGAACAATGACGACCAATAGCTC \\
& R-GCT GCTCATGCTTAGTCCAC \\
\hline
\end{tabular}

Abbreviations: $F$, forward; $R$, reverse. 
inhibitor (Anti-miR-NC) were obtained from RiboBio Co., Ltd (Guangzhou, China). TPC-1 cells in logarithmic phase were transfected with siRNAs $(50 \mathrm{nM})$ or mimics $(100 \mathrm{nM})$ and inhibitor (100 nM) using Lipofectamine 3000 (Thermo Fisher Scientific) following the manufacturer's instructions.

\section{Cell Counting Kit-8 (CCK-8) assay}

The CCK-8 assay was applied to measure cell proliferative ability according to the manufacturer's instruction. Briefly, transfected cells were seeded into plate wells at a density of $5 \times 10^{3}$ cells/well and incubated for 24-72 hours. At indicated times (24, 48, and 72 hours), $10 \mu \mathrm{L}$ of CCK-8 solution was added to each well and cultured for an additional for 4 hours. The absorbance was detected at $450 \mathrm{~nm}$ using an ELISA reader (Thermo Labsystems, Helsinki, Finland).

\section{Apoptosis detection by flow cytometry}

Cells were harvested 48 hours after transfection to detect cell apoptosis. Cell apoptosis was measured using the Annexin V-FITC Apoptosis Detection Kit (BD Biosciences, San Jose, CA, USA). The percentage of cell apoptosis was analyzed using a FACScalibur flow cytometer (Becton Dickinson, Franklin Lakes, NJ, USA) using CellQuestPro software (Becton Dickinson).

\section{Wound healing assay}

The migratory ability of cells was analyzed by a woundhealing assay at 24 hours after transfection. Briefly, transfected cells were cultured in six-well plates until full confluency. Subsequently, an artificial homogenous wound was created using a sterile plastic micropipette tip, followed by culture for 24 hours in serum-free DMEM medium (Thermo Fisher Scientific). The spread of the wound was observed and photographed at 0 and 24 hours after wounding using a light microscope (Olympus Corporation, Tokyo, Japan).

\section{Transwell invasion assay}

Transwell assay was performed to assess cell invasion at 24 hours after transfection. Briefly, transfected cells suspended in serum-free DMEM medium were added into the upper chambers pre-coated with Matrigel Matrix (BD Biosciences). Medium (600 $\mu \mathrm{L})$ supplemented with 10\% heat-inactivated FBS (Thermo Fisher Scientific) was placed into the lower chamber as a chemoattractant. After incubation for 24 hours at $37^{\circ} \mathrm{C}$, the cells that had invaded into the lower surface of the inserts were fixed with $4 \%$ paraformaldehyde and stained with $0.1 \%$ crystal violet, and counted within randomly selected five visual fields under a light microscope (×200 magnification; Olympus Corporation).

\section{Dual luciferase assay}

The binding sites between SNHG16 and miR-497 were predicted using Starbase v2.0 software (http://starbase. sysu.edu.cn/). SNHG16 fragments containing the putative miR-497 binding site (UGCUGCU) were chemically synthesized and inserted into a luciferase reporter vector (psiCHECK2; Promega Corporation, Madison, WI, USA) and designated as WT-SNHG16. The miR-497 binding site in the SNHG16 3' untranslated region (3'UTR) was mutated using the QuikChange site-directed mutagenesis kit (Stratagene, Cedar Creek, TX, USA) and named MT-SNHG16. For reporter assays, TPC-1 cells were co-transfected with WT-SNHG16 or MT-SNHG16 reporter plasmid and miR497 mimic or miR-NC using Lipofectamine 3000. At 48 hours after transfection, Firefly and Renilla luciferase activities in cell lysates were determined by the Dual Luciferase Reporter Assay system (Promega Corporation) according to the manufacturer's instruction. The relative luciferase activity was standardized with the Renilla luciferase activity.

\section{Statistical analysis}

All statistical analyses were performed using SPSS 19.0 (IBM Corporation, Armonk, NY, USA). All data were represented as mean \pm SD. Differences between two groups were analyzed by Student's $t$-test, and one-way ANOVA was applied to compare among more than two groups. The correlation between SNHG16 and miR-497 was evaluated using the Pearson's correlation analysis. In all cases, a $P$-value $<0.05$ was considered to indicate a statistically significant difference.

\section{Results}

\section{SNHG I 6 expression was upregulated in PTC tissues and cell lines}

To determine whether SNHG16 is dysregulated in PTC, qRT-PCR was conducted to determine the expression of SNHG16 in the PTC tissues and adjacent normal tissues of 48 patients with PTC. The data revealed that SNHG16 was dramatically higher in PTC tissues than in the normal thyroid tissues (Figure 1A, $P<0.01$ ). In addition, we also found that increased SNHG16 expression was closely associated with TNM stage and lymph node metastasis (Table 2), 

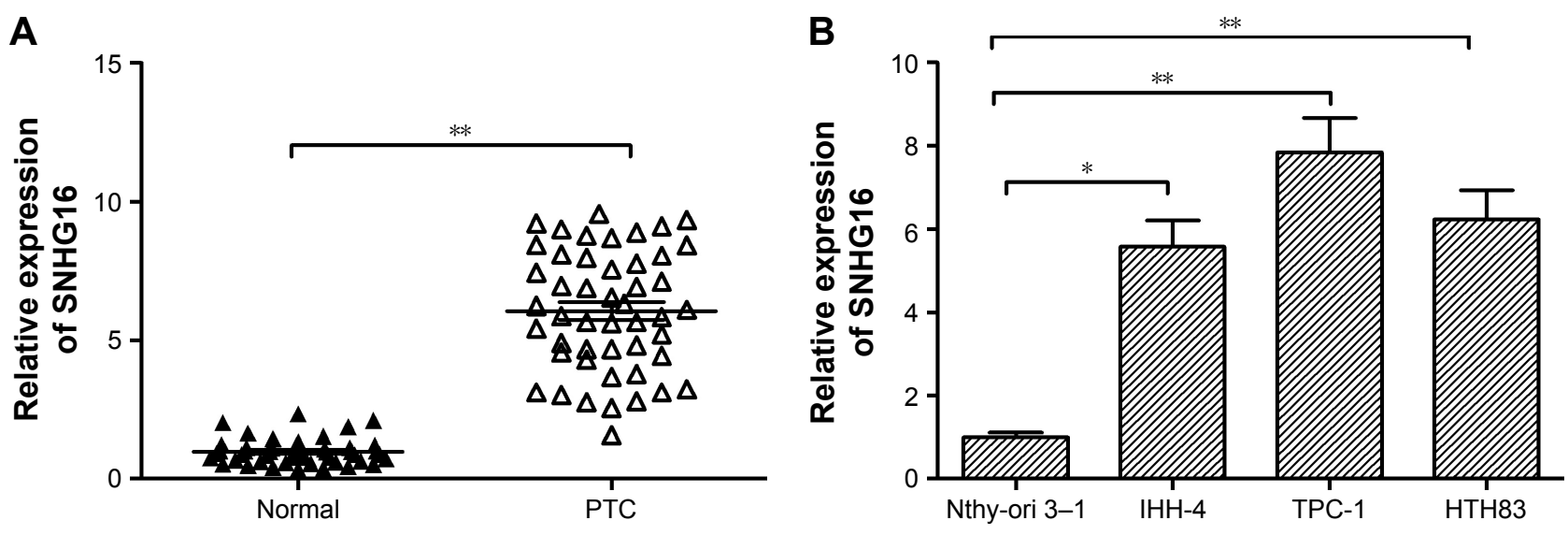

Figure I LncRNA SNHG 6 was significantly upregulated in PTC tissues and cell lines.

Notes: (A) The expression level of SNHGI6 was detected in human PTC and adjacent normal tissues by qRT-PCR. (B) SNHGI6 expression in three human PTC cell lines IHH-4, TPC-I, and HTH83, and normal thyroid follicular epithelial cell line Nthy-ori 3-I. The results represent the average of three independent experiments (mean \pm SD). $* P<0.05, * * P<0.01$.

Abbreviations: LncRNA, long noncoding RNA; PTC, papillary thyroid cancer; qRT-PCR, reverse-transcription quantitative PCR.

while there was no association with patient's age, gender, and tumor size $(P>0.05$; Table 2). To further support the abovementioned result, we examined the expression of SNHG16 in three PTC cell lines IHH-4, TPC-1, and HTH83, and normal thyroid follicular epithelial cell line. Our results also indicated that SNHG16 expression was significantly upregulated in PTC cell lines compared to that in Nthy-ori 3-1 cells (Figure 1B; $P<0.01$ ), in which TPC-1 cells exhibit the highest expression. Thus, TPC-1 cells were selected for the subsequent studies.

Table 2 Correlation between clinicopathological features and SNHG 16 expression in 48 patients with PTC

\begin{tabular}{|c|c|c|c|c|}
\hline \multirow[t]{2}{*}{ Variables } & \multirow[t]{2}{*}{$\begin{array}{l}\text { No of } \\
\text { cases }\end{array}$} & \multicolumn{2}{|c|}{\begin{tabular}{|l|} 
SNHG I6 \\
expression
\end{tabular}} & \multirow[t]{2}{*}{$P$-value } \\
\hline & & $\begin{array}{l}\text { High } \\
\text { (n [\%]) }\end{array}$ & $\begin{array}{l}\text { Low } \\
\text { (n [\%]) }\end{array}$ & \\
\hline Age (years) & & & & $P>0.05$ \\
\hline$<60$ & 22 & II (50.0) & II (50.0) & \\
\hline$\geq 60$ & 26 & $14(53.8)$ & $12(46.2)$ & \\
\hline Gender & & & & $P>0.05$ \\
\hline Male & 16 & $9(56.3)$ & $7(43.7)$ & \\
\hline Female & 32 & $16(50.0)$ & $16(50.0)$ & \\
\hline TNM stage & & & & $P<0.01$ \\
\hline I-II & 36 & $24(66.7)$ & $12(33.3)$ & \\
\hline III-IV & 12 & I (8.3) & II (9I.7) & \\
\hline Tumor size & & & & $P>0.05$ \\
\hline$<\mathrm{lcm}$ & 28 & $13(46.4)$ & $15(33.6)$ & \\
\hline$\geq 1 \mathrm{~cm}$ & 20 & $12(60.0)$ & $8(40.0)$ & \\
\hline Lymph node metastasis & & & & $P<0.01$ \\
\hline No & 35 & $23(65.7)$ & $12(34.3)$ & \\
\hline Yes & 13 & $2(15.4)$ & II (84.6) & \\
\hline
\end{tabular}

Abbreviation: PTC, papillary thyroid cancer.

\section{Knockdown of SNHGI 6 inhibited proliferation and promoted apoptosis in PTC cells}

To investigate the biological function of SNHG16 in PTC cell proliferation, siRNA against SNHG16 (si-SNHG16) was transfected into TPC-1 cells. Transfection efficiency was confirmed by qRT-PCR analysis (Figure 2A). CCK-8 assay demonstrated that silencing of SNHG16 significantly inhibited cell proliferation of TPC-1 cells in comparison to the cells of si-NC group (Figure 2B). Next, flow cytometry assay was performed to determine the effect of SNHG16 on cell apoptosis. We found that knockdown of SNHG16 significantly induced cell apoptosis in TPC-1 cells (Figure 2C, $P<0.01$ ).

\section{Knockdown of SNHG I 6 inhibited migration and invasion in PTC cells}

To clarify the role of SNHG16 in PTC metastasis, the present study analyzed the effects of SNHG16 on the migration and invasion of PTC cells. Using a wound-healing assay, a significantly decreased migration of TPC-1 cells transfected with the si-SNHG16 was observed compared with cells transfected with si-NC (Figure 3A). Similarly, an in vitro transwellinvasion assay demonstrated that knockdown of SNHG16 significantly inhibited invasion of TPC-1 cells (Figure 3B).

\section{The reciprocal interaction of SNHG 6 with miR-497 in PTC cells}

A growing body of evidence suggested that lncRNAs could serve as ceRNA or a molecular sponge in modulating the biological roles of microRNAs. ${ }^{20}$ Starbase 2.0 
A

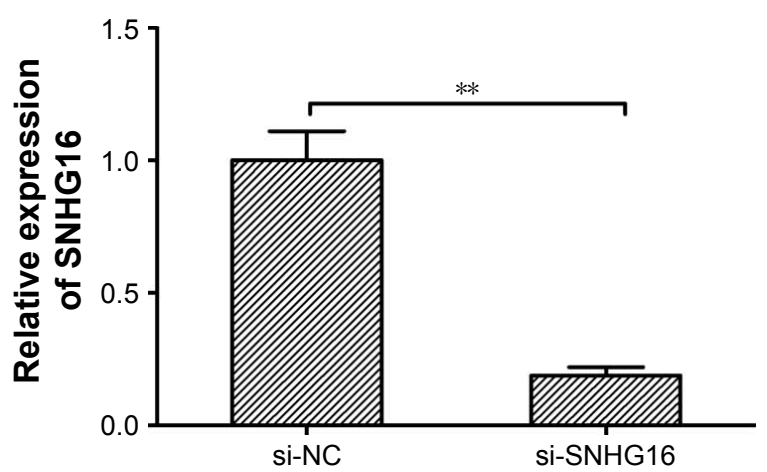

C

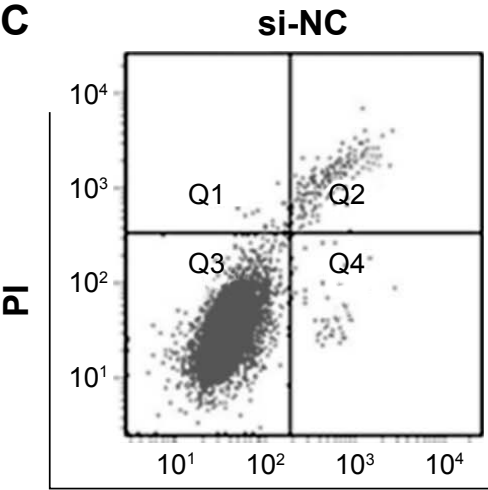

si-SNHG16

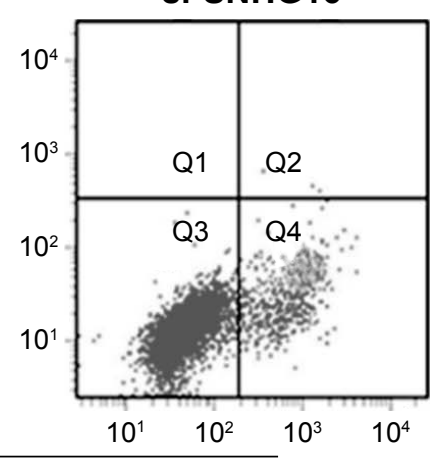

B

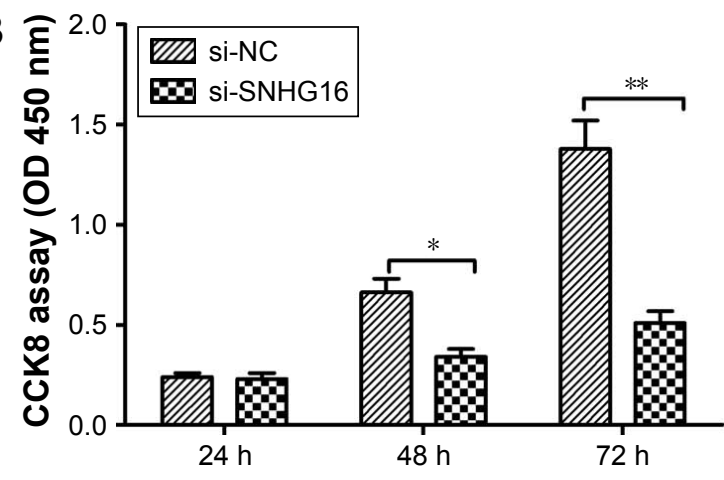

Annexin-V

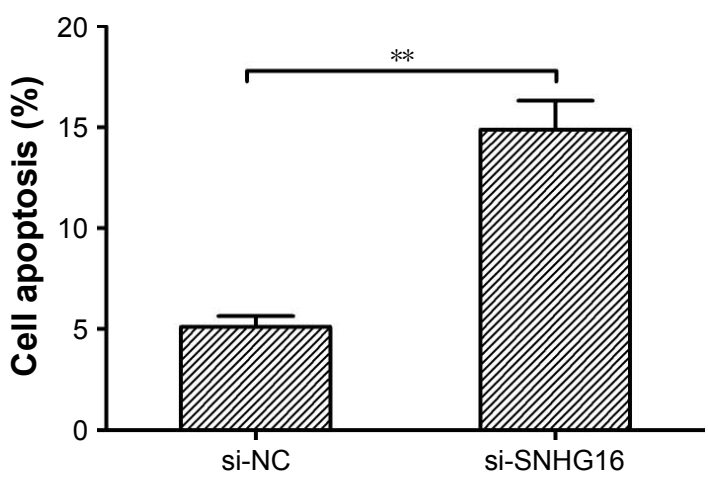

Figure 2 Knockdown of SNHGI6 inhibited cell proliferation and induced apoptosis in PTC cells.

Notes: (A) The expression level of SNHGI6 was examined in TPC-I cells transfected with si-SNHGI6 or si-NC. (B) Cell proliferation was measured in TPC-I cells transfected with si-SNHGI6 or si-NC by CCK-8 assay. (C) Cell apoptosis was examined in TPC-I cells transfected with si-SNHGI6 or si-NC by flow cytometry assay.

The results represent the average of three independent experiments (mean $\pm S D$ ). $* P<0.05, * * P<0.01$.

Abbreviations: PTC, papillary thyroid cancer; NC, negative control; CCK, Cell Counting Kit; PI, propidium iodide.

analysis revealed that miR-497 could bind with SNHG16 (Figure 4A). miR-497, a known tumor suppressor, has been reported to inhibit PTC progression by targeting multiple genes. To verify whether SNHG16 could bind with miR497 in PTC cells, a luciferase reporter assay was performed in TPC-1 cells, which revealed that overexpression of miR-497 clearly inhibited the luciferase activity of the wild-type SNHG16, whereas it had no influence on that of the mutant SNHG16 (Figure 4B). In addition, we also found that miR-497 overexpression markedly suppressed SNHG16 expression in TPC-1 cells, and transfection of miR-497 inhibitor increased SNHG16 expression in TPC-1 cells (Figure 4C). Meanwhile, silencing of SNHG16 significantly increased miR-497 expression in TPC-1 cells (Figure 4D). To further investigate the relationship between SNHG16 and miR-497 ex vivo, miR-497 expression levels were examined in PTC tissues and adjacent normal tissues. The result of qRT-PCR demonstrated that miR-497 expression was significantly downregulated in PTC tissues compared with that in adjacent normal tissues (Figure 4E).
Additionally, our result revealed that SNHG16 expression was inversely correlated with miR-497 expression levels in PTC tissues (Figure 4F).

\section{Inhibition of miR-497 partially rescues cells from the biological effects of SNHGI 6 in PTC cells}

To investigate whether SNHG16 functions by regulating miR-497, si-SNHG16, sh-NC, and si-SNHG16+ miR-497 inhibitor were separately transfected into TPC-1 cells. The results of qRT-PCR showed that miR-497 expression was increased in TPC-1 cells by transfection with si-SNHG16, while miR-497 expression was decreased in TPC-1 cells by transfection with miR-497 inhibitor (Figure 5A). Moreover, our results demonstrated that inhibition of miR-497 partially abrogated the effect of SNHG16 whose levels depleted on cell proliferation, apoptosis, migration, and invasion in TPC-1 cells (Figure 5B-E). Taken together, these results implied that miR-497 might be involved in the SNHG16mediated oncogenic activity. 
A
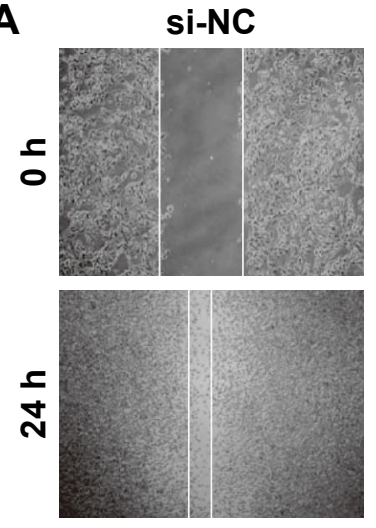

B
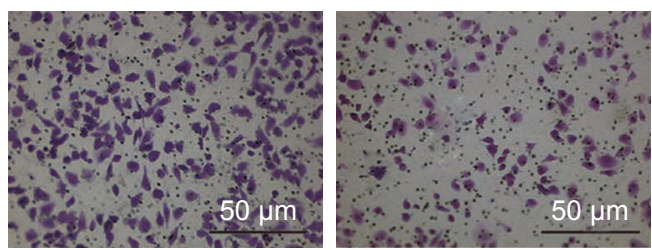

si-SNHG16
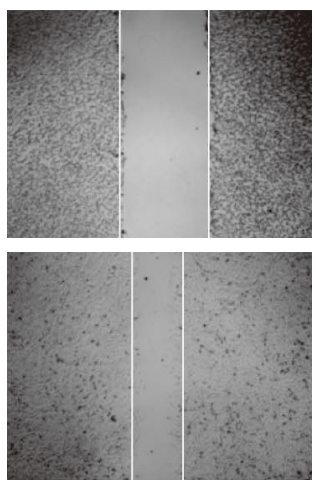
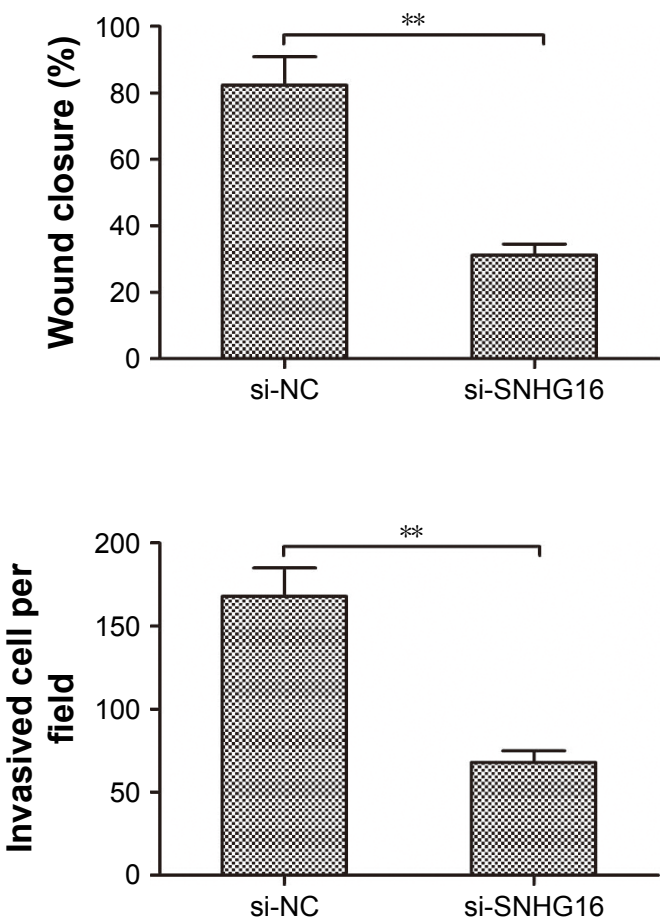

Figure 3 Knockdown of SNHGI6 inhibited cell migration and invasion in PTC cells.

Notes: (A) Cell migration was measured in TPC-I cells transfected with si-SNHGI6 or si-NC by wound-healing assay. (B) Cell invasion was examined in TPC-I cells transfected with si-SNHGI6 or si-NC by transwell invasion assay. The results represent the average of three independent experiments (mean \pm SD). $* * P<0.01$.

Abbreviations: PTC, papillary thyroid cancer; NC, negative control.

\section{SNHG I6 regulated the expression of downstream targets of miR-497 in PTC cells}

Previous reports showed that brain-derived neurotrophic factor (BDNF) and yes-associated protein 1 (YAP1) are targets of miR-497 in PTC cells. ${ }^{22,23}$ Further, we were interested in determining whether SNHG16 regulated these two targets of miR-497 as well. We found that knockdown of miR-497 significantly downregulated the expression of BDNF and YAP1, whereas miR-497 inhibitor partially abolished the effect of SNHG16 in TPC-1 cells (Figure 6A and B). In addition, SNHG16 expression was positively correlated with BDNF and YAP1 in PTC tissues (Figure 6C and D). Taken together, SNHG16 knockdown suppressed the downstream targets via sponging miR-497 in PTC cells.

\section{Discussion}

Growing evidence has indicated that dysregulation of lncRNAs plays crucial roles in tumorigenesis and metastasis in PTC; $; 10$ thus, this has attracted interest from numerous researchers hoping to find novel lncRNAs involved in PTC progression. In this study, we demonstrated that the level of SNHG16 was significantly upregulated in PTC tissues and cell lines, and that increased SNHG16 was statistically associated with advanced TNM stage and lymph node metastasis. To further investigate the clinical significance of SNHG16, sufficient PTC samples were needed. Furthermore, the biological function of SNHG16 was explored in vitro by a series of molecular experiments. The results demonstrated that knockdown of SNHG16 in PTC cells significantly inhibited cell proliferation, induced cell apoptosis, and suppressed migration and invasion. Thus, the abovementioned data indicated that SNHG16 might play a fundamental role in PTC progression.

SNHG16, a signal transducer and activator of transcription 3 (STAT3) modulator, ${ }^{13,24}$ has been reported to be upregulated and function as an oncogene in multiple types of cancer. ${ }^{11-18,24,25}$ However, the role of SNHG16 in the PTC remained largely unclear. In the present study, we evaluated the expression of SNHG16 and its relationship with the clinical features in patients with PTC, and found that SNHG16 expression was significantly upregulated in PTC tissues and cell lines, and that increased SNHG16 expression was closely associated with TNM stage and lymph node metastasis. To investigate the biological roles of SNHG16 in PTC cells, the loss-of-function assay was performed by silencing SNHG16. 
A

WT-SNHG16 $5^{\prime} \ldots$ ACAGCCCUGCCUCUGCUGCUA... $3^{\prime}$ miR-497 3' UGUUUGGUGUCACACGACGAC $5^{\prime}$

MT-SNHG16 $\quad 5^{\prime} \ldots$...ACAGCCCUGCCUCACGACGAA.... $3^{\prime}$ mutant sites

C

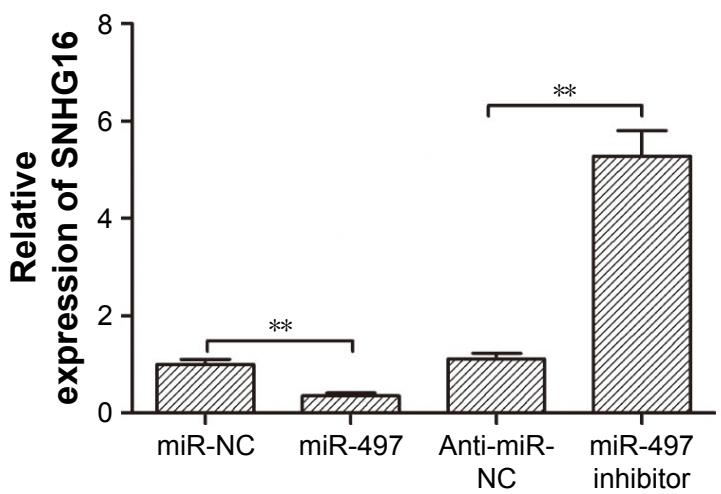

E

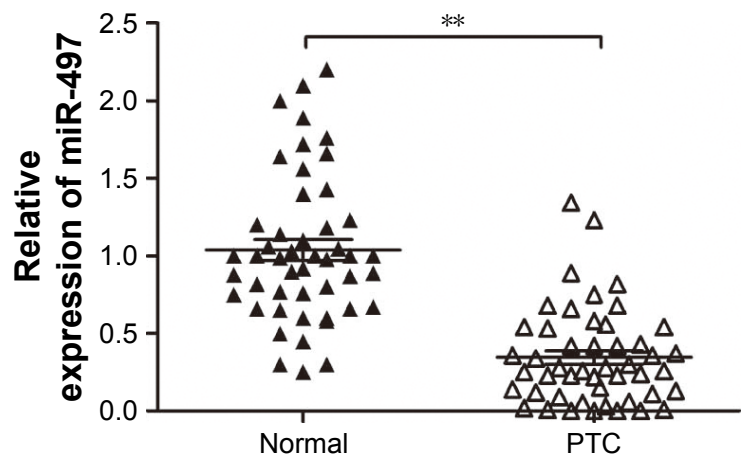

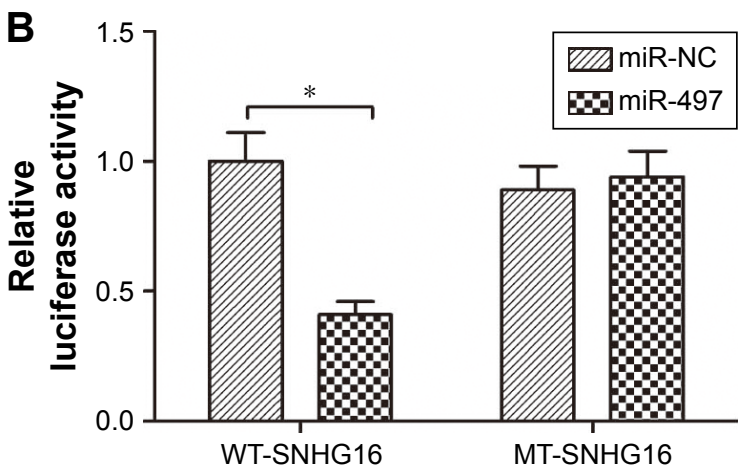

D

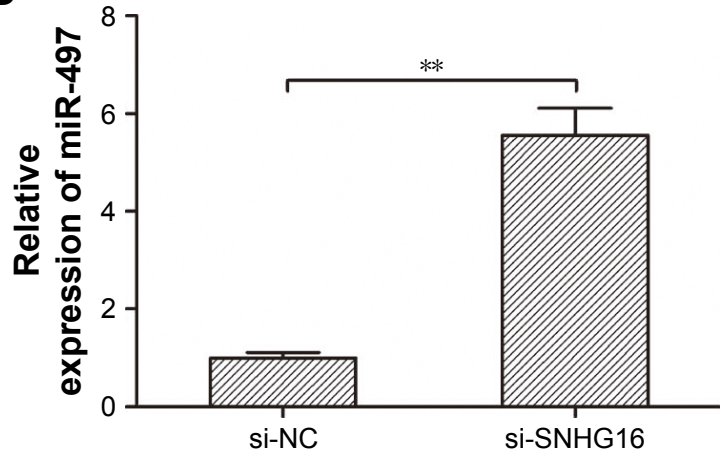

$\mathbf{F}$

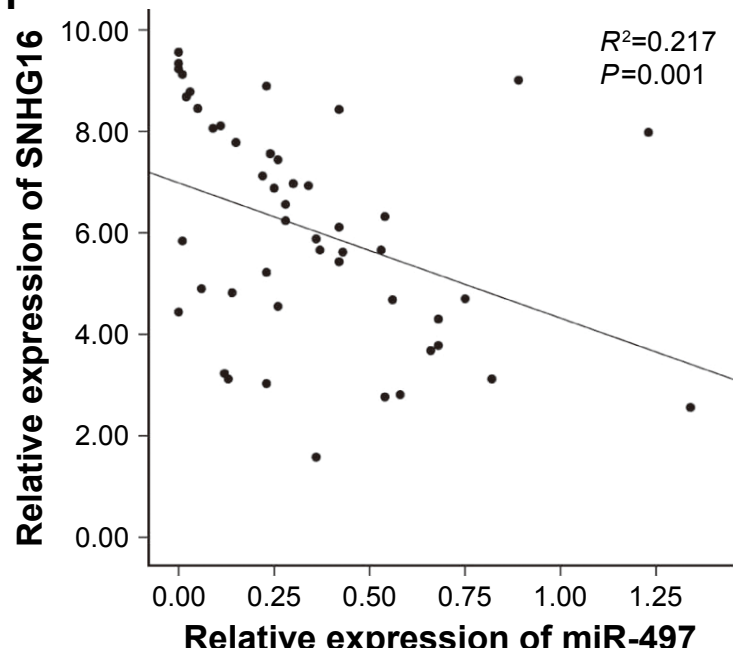

Figure 4 SNHGI 6 is a direct target of miR-497 in PTC cells.

Notes: (A) The binding sites of SNHGI6 (WT-SNHG I6) and mutants sites (MT-SNHG I6) are shown. (B) Relative luciferase activity was detected in TPC-I cells transfected with WT-SNHGI6/MT-SNHGI6 and miR-497 mimic/miR-NC. (C) The expression of SNHGI6 was examined in TPC-I cells transfected with miR-497 mimics, miR-NC, miR-497 inhibitor, and anti-miR-NC by qRT-PCR. (D) The expression of miR-497 was measured in TPC-I cells transfected with si-SNHGI6 and si-NC by qRT-PCR. (E) The expression level of SNHGI6 was detected in human PTC and adjacent normal tissues by qRT-PCR. (F) The correlation between miR-497 and SNHGI6 expression in PTC tissues ( $n=48$ ) by Pearson's analysis. The results represent the average of three independent experiments (mean \pm SD). $* P<0.05, * * P<0.01$.

Abbreviations: PTC, papillary thyroid cancer; NC, negative control; WT, wild type; MT, mutant type; qRT-PCR, reverse-transcription quantitative PCR.

Our results showed that knockdown of SNHG16 significantly inhibited PTC cell proliferation, migration, and invasion, as well as induced cell apoptosis. Our findings indicated that SNHG16 functioned as an oncogene in PTC progression.

Increasing evidence has been suggested that IncRNA could function as ceRNAs that act as molecular sponges for miRNAs to reduce the expression and activity of target
miRNAs. ${ }^{26}$ SNHG16 has been reported to serve as a ceRNA in several cancers by sponging multiple miRNAs, including miR-98, ${ }^{13}$ miR-140-5 p ${ }^{11}$ miR-520d-3p, ${ }^{24}$ miR-20a-3p,${ }^{17}$ miR-4518, ${ }^{27}$ miR-216-5p, ${ }^{12}$ and miR-15a. ${ }^{28}$ Here, bioinformatics analysis was conducted to identify the miRNAs that can bind to complementary sequences in SNHG16. We found that miR-497 shares the complementary binding sites 

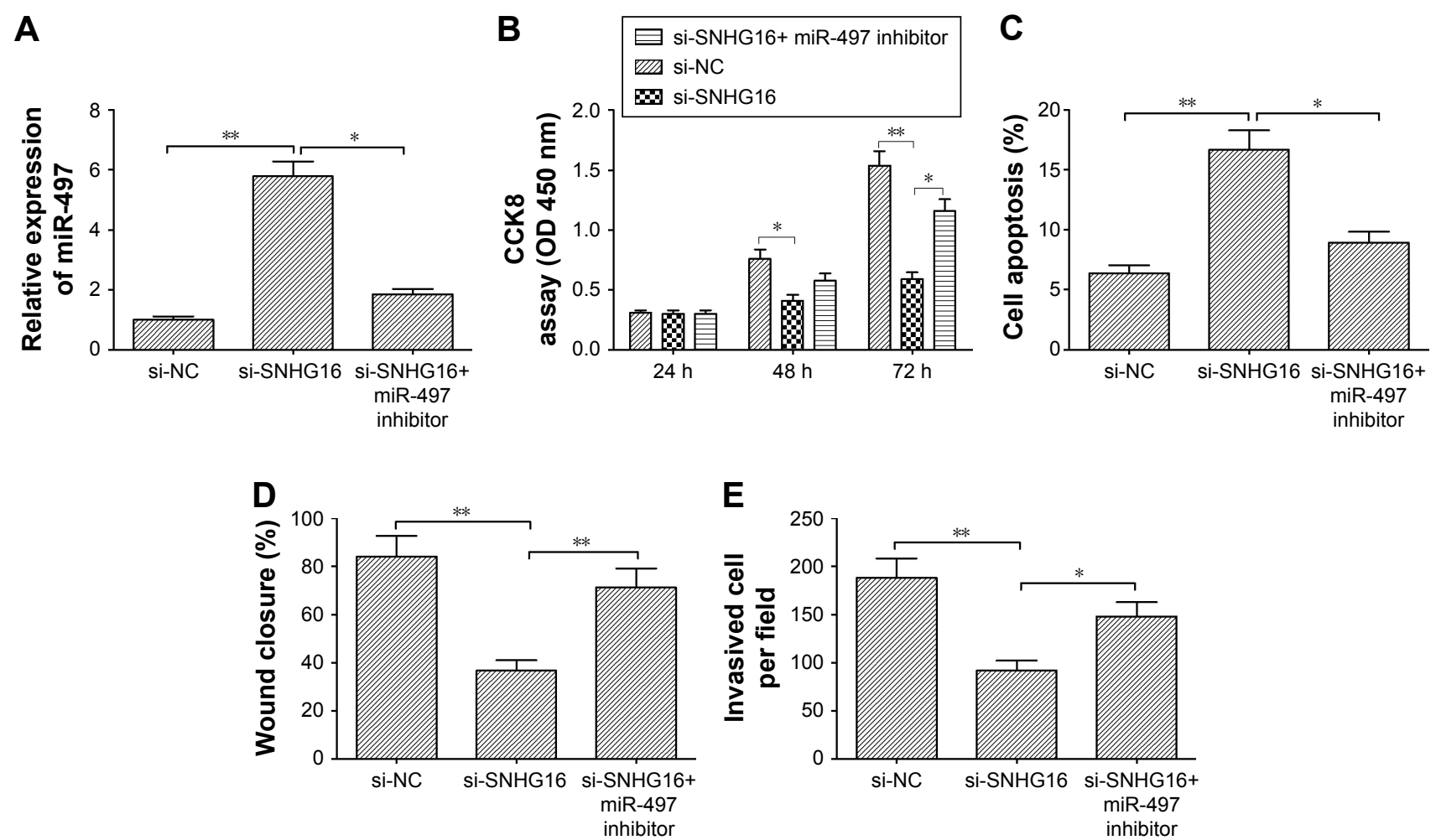

Figure 5 Inhibition of miR-497 partially rescues cells from the biological effects of SNHGI6 in PTC cells.

Notes: (A) The expression of miR-497 expression was examined in TPC-I cells transfected with si-NC, si-SNHGI6, and si-SNHGI6+ miR-497 inhibitor. (B-E) Cell proliferation, apoptosis, migration, and invasion were determined in TPC-I cells transfected with si-NC, si-SNHGI6, and si-SNHGI6+ miR-497 inhibitor. The results represent the average of three independent experiments (mean $\pm S D$ ). $* P<0.05$, $* * P<0.01$.

Abbreviations: PTC, papillary thyroid cancer; NC, negative control; CCK, Cell Counting Kit.

at $3^{\prime}$-UTR, which was confirmed by the luciferase assay. miR-497, a known tumor suppressor, was reported to suppress PTC growth in vitro and in vivo by targeting BDNF and YAP1. ${ }^{22,23}$ The present study demonstrated that transfection of miR-497 significantly decreased SNHG16 expression, and transfection with miR-497 inhibitor increased SNHG16 expression in TPC-1 cells. Moreover, SNHG16 knockdown obviously increased miR-497 expression in TPC-1 cells.
The present study also showed that miR-497 expression was decreased in PTC tissues, and its expression was inversely correlated with SNHG16 in PTC tissues. Importantly, miR-497 inhibitor reversed the inhibitory effect of SNHG16 knockdown on cell proliferation, apoptosis, migration, and invasion. In addition, we investigated whether SNHG16 regulated these two targets of miR-497 as well. We found that knockdown of miR-497 significantly downregulated the
A

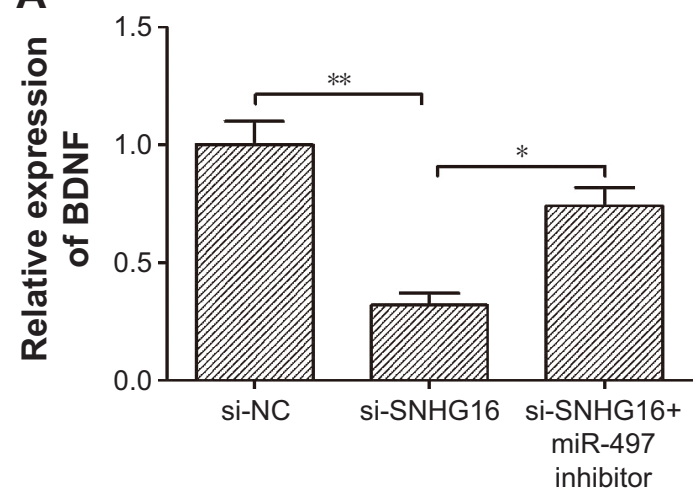

B

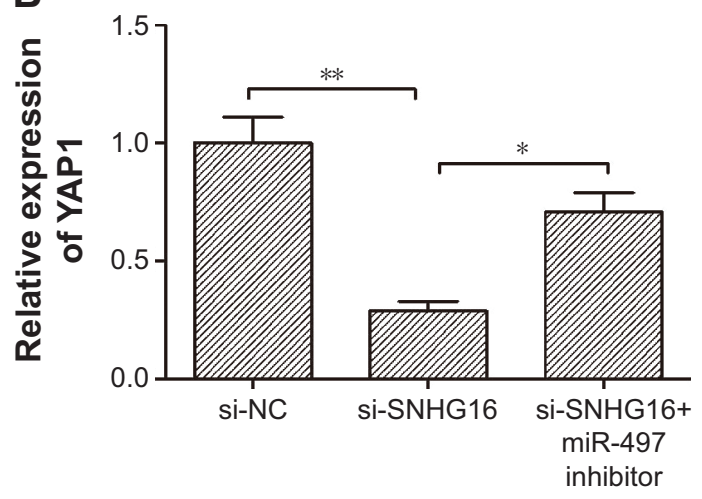

Figure 6 (Continued) 

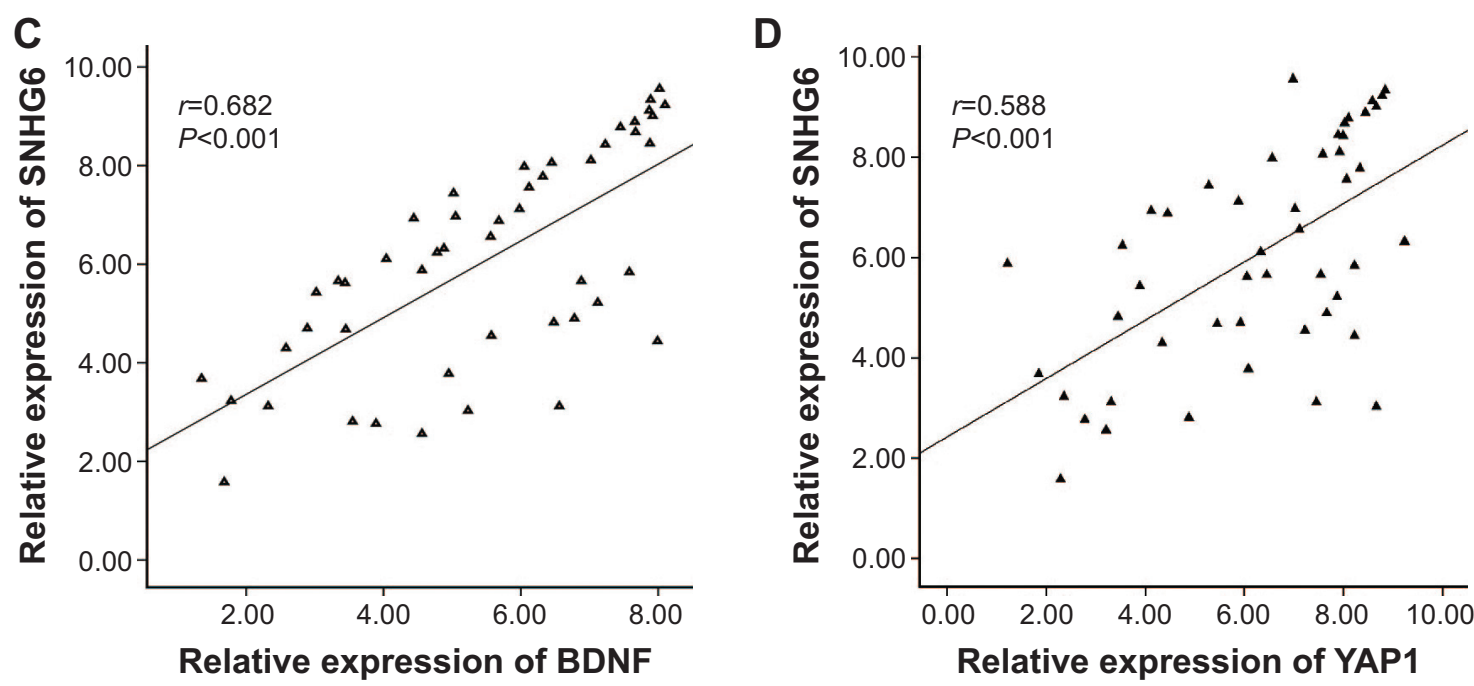

Figure 6 SNHG 16 regulated the expression of downstream targets of miR-497 in PTC cells.

Notes: (A and B) The expression of BDNF and YAPI was measured in TPC-I cells transfected with sh-NC or si-SNHGI6, with or without the miR-497 inhibitor. (C) A positive association between BDNF mRNA and SNHGI 6 expression in PTC tissues was identified by Pearson's correlation analysis. (D) A positive association between YAPI mRNA and SNHGI6 expression in PTC tissues was identified by Pearson's correlation analysis. The results represent the average of three independent experiments (mean $\pm \mathrm{SD}$ ). $* P<0.05, * * P<0.01$.

Abbreviation: PTC, papillary thyroid cancer.

expression of BDNF and YAP1, whereas miR-497 inhibitor partially abolished the effect of SNHG16 in TPC-1 cells. SNHG16 expression was positively correlated with BDNF and YAP1 in PTC tissues. Taken together, these findings suggested that SNHG16 exerts physiological functions in PTC via sponging miR-497.

\section{Conclusion}

Taken together, the findings revealed that SNHG16 expression level was increased in PTC tissues and cell lines, and increased SNHG16 levels were positively associated with advanced TNM stage and lymph node metastasis. The results also revealed that knockdown of SNHG16 inhibited PTC cell proliferation, migration, and invasion by regulating miR497. These results suggested that SNHG16 might represent a novel therapeutic strategy for treating patients with PTC.

\section{Acknowledgment}

This research was supported by the Research Fund of Direct Health Project in Jilin Province (Grant number: SCZSY201712).

\section{Disclosure}

The authors report no conflicts of interest in this work.

\section{References}

1. Torre LA, Bray F, Siegel RL, Ferlay J, Lortet-Tieulent J, Jemal A. Global cancer statistics, 2012. CA Cancer J Clin. 2015;65(2):87-108.
2. Ferlay J, Shin HR, Bray F, Forman D, Mathers C, Parkin DM. Estimates of worldwide burden of cancer in 2008: GLOBOCAN 2008. Int J Cancer. 2010;127(12):2893-2917.

3. Xing M. Molecular pathogenesis and mechanisms of thyroid cancer. Nat Rev Cancer. 2013;13(3):184-199.

4. Lundgren CI, Hall P, Dickman PW, Zedenius J. Clinically significant prognostic factors for differentiated thyroid carcinoma: a populationbased, nested case-control study. Cancer. 2006;106(3):524-531.

5. Cech TR, Steitz JA. The noncoding RNA revolution-trashing old rules to forge new ones. Cell. 2014;157(1):77-94.

6. Ponting CP, Oliver PL, Reik W. Evolution and functions of long noncoding RNAs. Cell. 2009;136(4):629-641.

7. Sun T. Long noncoding RNAs act as regulators of autophagy in cancer. Pharmacol Res. 2018;129:151-155.

8. Huarte M. The emerging role of lncRNAs in cancer. Nat Med. 2015; 21(11):1253-1261.

9. Jing W, Li X, Peng R, et al. The diagnostic and prognostic significance of long noncoding RNAs expression in thyroid cancer: A systematic review and meta-analysis. Pathol Res Pract. 2018;214(3):327-334.

10. Murugan AK, Munirajan AK, Alzahrani AS. Long noncoding RNAs: emerging players in thyroid cancer pathogenesis. Endocr Relat Cancer. 2018;25(2):R59-R82.

11. Zhang K, Chen J, Song H, Chen LB. SNHG16/miR-140-5p axis promotes esophagus cancer cell proliferation, migration and EMT formation through regulating ZEB1. Oncotarget. 2018;9(1):1028-1040.

12. Zhu H, Zeng Y, Zhou CC, Ye W. SNHG16/miR-216-5p/ZEB1 signal pathway contributes to the tumorigenesis of cervical cancer cells. Arch Biochem Biophys. 2018;637:1-8.

13. Feng F, Chen A, Huang J, Xia Q, Chen Y, Jin X. Long noncoding RNA SNHG16 contributes to the development of bladder cancer via regulating miR-98/STAT3/Wnt/B-catenin pathway axis. J Cell Biochem. 2018;119(11):9408-9418.

14. Yang BY, Meng Q, Sun Y, Gao L, Yang JX. Long non-coding RNA SNHG16 contributes to glioma malignancy by competitively binding miR20a-5p with E2F1. J Biol Regul Homeost Agents. 2018;32(2):251-261.

15. Yang XS, Wang GX, Luo L. Long non-coding RNA SNHG16 promotes cell growth and metastasis in ovarian cancer. Eur Rev Med Pharmacol Sci. 2018;22(3):616-622. 
16. Lian D, Amin B, Du D, Yan W. Enhanced expression of the long noncoding RNA SNHG16 contributes to gastric cancer progression and metastasis. Cancer Biomark. 2017;21(1):151-160.

17. Cai C, Huo Q, Wang X, Chen B, Yang Q. SNHG16 contributes to breast cancer cell migration by competitively binding miR-98 with E2F5. Biochem Biophys Res Commun. 2017;485(2):272-278.

18. Christensen LL, True K, Hamilton MP, et al. SNHG16 is regulated by the Wnt pathway in colorectal cancer and affects genes involved in lipid metabolism. Mol Oncol. 2016;10(8):1266-1282.

19. Tian S, Rulli S, Fahey TJ, Lader E. Abstract 531: lncRNA profiling and its potential usage as a thyroid cancer biomarker. Cancer Res. 2015;75(15 Suppl):531.

20. Tay Y, Rinn J, Pandolfi PP. The multilayered complexity of ceRNA crosstalk and competition. Nature. 2014;505(7483):344-352.

21. Lee H, Park JE, Nam JM. Bio-barcode gel assay for microRNA. Nat Commun. 2014;5:3367.

22. Cheng H, Dong H, Feng J, Tian H, Zhang H, Xu L. miR-497 inhibited proliferation, migration and invasion of thyroid papillary carcinoma cells by negatively regulating YAP1 expression. Onco Targets Ther. 2018;11:4711-4721.
23. Wang $\mathrm{P}$, Meng X, Huang $\mathrm{Y}$, et al. MicroRNA-497 inhibits thyroid cancer tumor growth and invasion by suppressing BDNF. Oncotarget. 2017;8(2):2825-2834.

24. Zhao W, Fu H, Zhang S, Sun S, Liu Y. LncRNA SNHG16 drives proliferation, migration, and invasion of hemangioma endothelial cell through modulation of miR-520d-3p/STAT3 axis. Cancer Med. Epub 2018 May 29.

25. Han GH, Lu KJ, Wang P, Ye J, Ye YY, Huang JX. LncRNA SNHG16 predicts poor prognosis in ESCC and promotes cell proliferation and invasion by regulating Wnt/ $\beta$-catenin signaling pathway. Eur Rev Med Pharmacol Sci. 2018;22(12):3795-3803.

26. Qi X, Zhang DH, Wu N, Xiao JH, Wang X, Ma W. ceRNA in cancer: possible functions and clinical implications. J Med Genet. 2015;52(10): 710-718.

27. Lu YF, Cai XL, Li ZZ, et al. LncRNA SNHG16 Functions as an Oncogene by Sponging MiR-4518 and Up-Regulating PRMT5 Expression in Glioma. Cell Physiol Biochem. 2018;45(5):1975-1985.

28. Wang W, Lou C, Gao J, Zhang X, Du Y. LncRNA SNHG16 reverses the effects of miR-15a/16 on LPS-induced inflammatory pathway. Biomed Pharmacother. 2018;106:1661-1667.
OncoTargets and Therapy

\section{Publish your work in this journal}

OncoTargets and Therapy is an international, peer-reviewed, open access journal focusing on the pathological basis of all cancers, potential targets for therapy and treatment protocols employed to improve the management of cancer patients. The journal also focuses on the impact of management programs and new therapeutic agents and protocols on

\section{Dovepress}

patient perspectives such as quality of life, adherence and satisfaction The manuscript management system is completely online and includes a very quick and fair peer-review system, which is all easy to use. Visit http://www.dovepress.com/testimonials.php to read real quotes from published authors. 